\title{
Infrared extrapolations for atomic nuclei
}

\author{
R. J. Furnstahl ${ }^{1}$, G. Hagen ${ }^{2,3}$, T. Papenbrock ${ }^{3,2}$, and \\ K. A. Wendt ${ }^{3,2}$ \\ ${ }^{1}$ Department of Physics, The Ohio State University, Columbus, OH 43210 \\ ${ }^{2}$ Physics Division, Oak Ridge National Laboratory, Oak Ridge, TN 37831 USA \\ ${ }^{3}$ Department of Physics and Astronomy, University of Tennessee, Knoxville, TN \\ 37996, USA \\ E-mail: furnstahl.1@osu.edu, hageng@ornl.gov, tpapenbr@utk.edu, \\ kwendt2@utk.edu
}

\begin{abstract}
Harmonic oscillator model-space truncations introduce systematic errors to the calculation of binding energies and other observables. We identify the relevant infrared scaling variable and give values for this nucleus-dependent quantity. We consider isotopes of oxygen computed with the coupled-cluster method from chiral nucleon-nucleon interactions at next-to-next-to-leading order and show that the infrared component of the error is sufficiently understood to permit controlled extrapolations. By employing oscillator spaces with relatively large frequencies, well above the energy minimum, the ultraviolet corrections can be suppressed while infrared extrapolations over tens of $\mathrm{MeVs}$ are accurate for ground-state energies. However, robust uncertainty quantification for extrapolated quantities that fully accounts for systematic errors is not yet developed.
\end{abstract}

PACS numbers: 21.10.Dr, 21.60.-n, 31.15.Dv, 21.30.-x

Submitted to: J. Phys. G: Nucl. Phys. 


\section{Introduction}

Wave-function based methods for computing atomic nuclei, such as exact diagonalization [1, 2], coupled cluster [3, 4, 5], self-consistent Green's functions [6], or in-medium similarity renormalization group (SRG) [7, 8], require a computational effort that depends strongly on the size of the Hilbert space used. Truncating this model space introduces systematic errors that must be quantitatively analyzed for a given Hamiltonian and nucleus. By understanding these errors, we can devise controlled extrapolation techniques for energies and other observables.

Though we deal with a quantum mechanical problem, it is most instructive to look first at classical phase space. Let us consider the deuteron wave function, calculated from a realistic two-body interaction, and compute its Wigner transform. We recall that the Wigner transform

$$
f(\boldsymbol{r}, \boldsymbol{p})=\int d^{3} \boldsymbol{k} \frac{e^{\imath \boldsymbol{r} \cdot \boldsymbol{k}}}{(2 \pi)^{3}} \psi^{*}(\boldsymbol{p}+\boldsymbol{k} / 2) \psi(\boldsymbol{p}-\boldsymbol{k} / 2),
$$

is a mapping of a wave function to a phase-space distribution. Figure 1 shows the results for chiral effective field theory (EFT) Hamiltonians [9] with two different cutoffs. We see that the dominant part of the Wigner transform extends in (radial) position essentially up to the deuteron radius (though the exponential tail casts a long shadow), and in momentum space up to the cutoff of the interaction.

Generalizing this result, the number of single-particle states required to compute a nucleus with radius $R$ from an interaction with cutoff $\Lambda$ is essentially given by

$$
n=\frac{1}{(2 \pi)^{3}} \int^{R} d^{3} r \int^{\Lambda} d^{3} p \propto(R \Lambda)^{3} .
$$

Here, we have not counted spin/isospin degrees of freedom. The key result is that the number of single-particle states grows as the third power of the cutoff, and it is proportional to $R^{3} \propto A$ for a nucleus with mass number $A$. The proportionality constant in Eq. (2) depends on the actual basis one uses, and some efficiencies can possibly be gained from abandoning the oscillator basis in favor of Berggren bases [10], Sturmian bases [11] or discrete variable representations [12]. Equation (2) also makes clear that halo nuclei can be as expensive to compute as much heavier nuclei, and that much is to be gained by lowering the cutoff $\Lambda$ of the interaction. This latter point explains why low-momentum interactions (e.g. $V_{\text {low } k}$ [13] or SRG [14]) are so popular.

When working with the harmonic-oscillator basis, its extent in position and momentum space must exceed the radius of the nucleus and the cutoff of the interaction, respectively. In other words, one must choose the oscillator frequency and the maximum excitation energy such that the ellipsoidal phase-space area of the oscillator covers the relevant parts of the Wigner function for the nucleus. Let $b=\hbar / \sqrt{m \hbar \Omega}$ denote the oscillator length, where $m$ is the nucleon mass. Then a simple semiclassical argument implies that for an oscillator basis with up to $N$ excited quanta, we must demand

$$
\sqrt{2 N} \geq R / b \quad \text { and } \quad \sqrt{2 N} \geq \Lambda b .
$$


The ellipses corresponding to equality signs in these expressions are also shown in Fig. 1. We see that only the tails of the Wigner function - both in the directions of position and momentum - extend beyond the ellipses. Let us also note that Fig. 1 serves mainly as an illustration but should not be used for quantitative conclusions. Only in the semiclassical limit is it possible to quantitatively relate Wigner functions to classical phase-space structures [15]. The questions to address are what error results from the omission of these tails for the binding energy and other observables in a finite oscillator basis, and how quickly do these quantities converge as the number of basis states is increased?
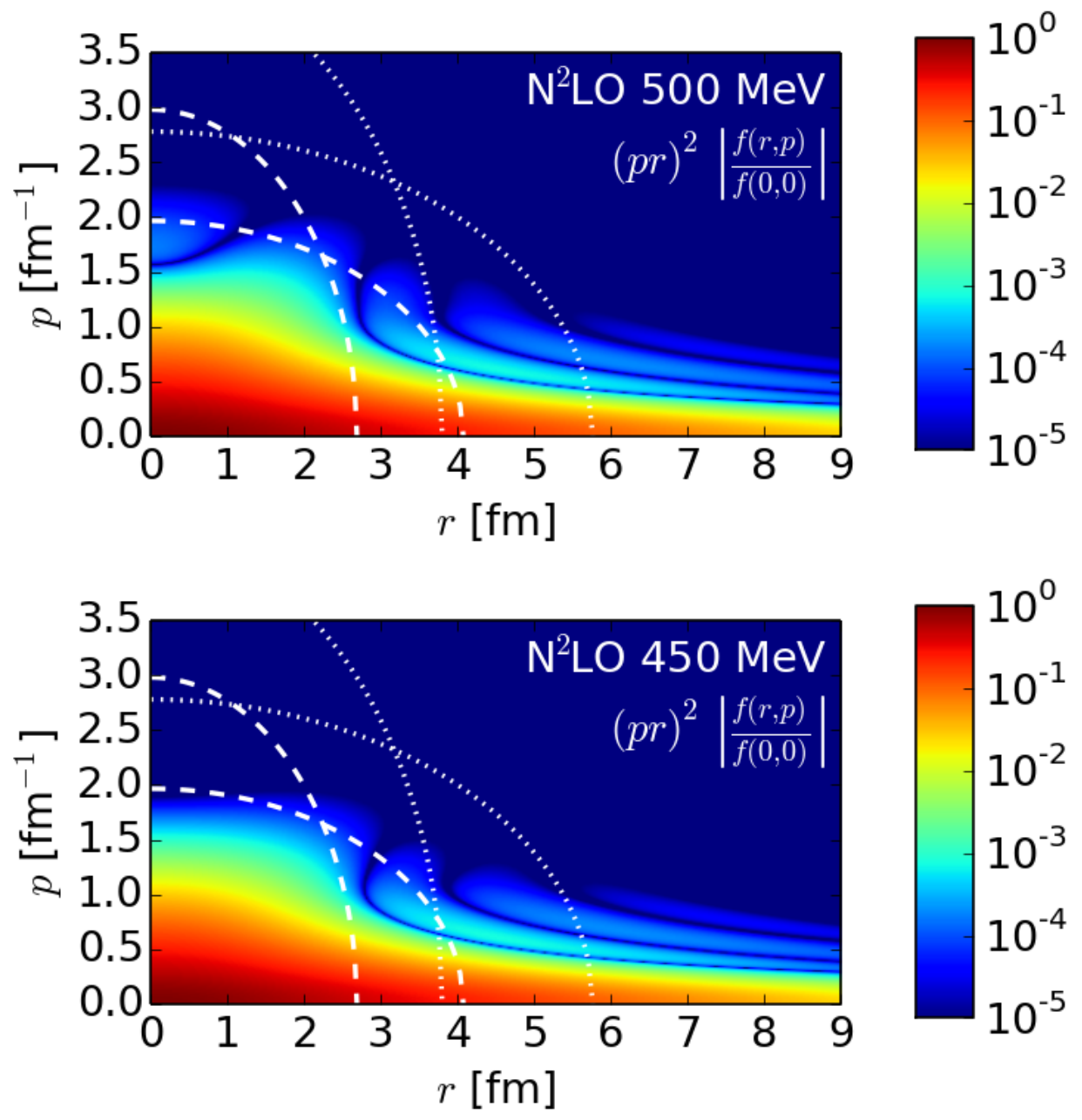

Figure 1. (Color online) Top: "s-wave" projection of Wigner function from deuteron wave function for a chiral $\mathrm{N}^{2} \mathrm{LO}$ potential with a regulator cutoff of $\Lambda_{\mathrm{UV}}=500 \mathrm{MeV}$. Abscissa is $r$ in units of $\mathrm{fm}$, ordinate is momentum $p$ in units of $\mathrm{fm}^{-1}$. The dashed lines are semi-classical harmonic oscillator cutoffs for $N=4$ and two different oscillator frequencies $(\hbar \Omega=20$ and $50 \mathrm{MeV})$. The dotted lines are the same with $N=8$. Bottom: same as top but for a lower regulator cutoff $\Lambda_{\mathrm{UV}}=450 \mathrm{MeV}$. 


\section{Infrared cutoffs of the oscillator basis}

The semi-classical estimates (3) are a useful guide, but for practical purposes we have to understand how the infrared (IR) cutoff $\Lambda_{\mathrm{IR}} \equiv \pi / L$ and ultraviolet (UV) cutoff $\Lambda_{\mathrm{UV}}$ of a finite oscillator basis can become tools for the extrapolation of observables. The leading-order estimates for these cutoffs are based on $L \approx \sqrt{2 N} b$ and $\Lambda_{\mathrm{UV}} \approx \sqrt{2 N} / b$, respectively [16, 17, 18]. Clearly, $L$ and $\Lambda_{\mathrm{UV}}$ are semiclassical estimates for the extent of the oscillator basis in position and momentum space, respectively.

The nucleon-nucleon forces (and many-body forces) used for wave-function based methods, such as chiral effective field theory (EFT) or low-momentum interactions, all have momentum-space regulators that rapidly drive them to zero above a cutoff $\Lambda$. If $\Lambda_{\mathrm{UV}}$ is less than or comparable to $\Lambda$, the UV corrections to the energy and other observables will depend on the details of the regulators (but less on the nucleus under consideration). But when $\Lambda_{\mathrm{UV}}$ sufficiently exceeds $\Lambda$ (see Sec. 4), rapid UV convergence is observed in practical calculations, so that UV extrapolations are not needed.

In this paper, we focus solely on IR extrapolations. We note that the long wavelength structure of a bound state stems from its exponential fall-off in position space and is independent of the details of the interaction. A finite extent of the oscillator basis in position space cuts off this exponential tail and thereby yields an energy correction. References [19, 20, 21] provide a theoretical basis for IR extrapolations. It is established in these papers that a finite oscillator basis with $N$ excited quanta and oscillator length $b$ is, at low momenta, indistinguishable from a box of size

$$
L_{2}(N, \hbar \Omega) \approx \sqrt{2(N+3 / 2+2)} b .
$$

Here, the approximate sign indicates that this is the next-to-leading order approximation of the box size in the limit of $N \gg 1$. This result can be derived from the fact that $\Lambda_{\mathrm{IR}}^{2} \equiv\left(\pi / L_{2}\right)^{2}$ is the lowest eigenvalue of the momentum operator squared in the finite oscillator basis [20]. A single-particle wave function of an $s$-wave bound state, approximated in a finite oscillator basis, only differs by high-momentum components from the same wave function approximated in a spherical well of radius $L_{2}$. For a partial wave with angular momentum $l$, one has $N=2 n+l$ in Eq. (4). Thus, for fixed oscillator spacing $\hbar \Omega$ the length $L_{2}(N, \hbar \Omega)$ is a staircase function that increases at even (odd) values of $N$ for even (odd) values of $l$.

This knowledge can be used to understand the IR extrapolations of bound states. The equivalent finite size $L_{2}$ of the oscillator basis has the effect on a position-space bound state of enforcing that the wave function has a node at $L_{2}$. This correction to the true bound-state wave function is a long-wavelength phenomenon and can thus be understood in model-independent ways [20, 21]. The leading-order IR extrapolation formula for an $s$-wave bound-state energy of a single-particle system is

$$
E\left(L_{2}\right)=E_{\infty}+\frac{\hbar^{2} k_{\infty} \gamma_{\infty}^{2}}{\mu} \exp \left(-2 k_{\infty} L_{2}\right)
$$

Here, $k_{\infty}$ is the bound-state momentum, i.e. $E_{\infty}=\hbar^{2} k_{\infty}^{2} /(2 \mu)$ is the bound-state energy, $\mu$ is the reduced mass, and $\gamma_{\infty}$ is the asymptotic normalization constant. Higher-order 
corrections, which are suppressed by powers of $\exp \left(-2 k_{\infty} L_{2}\right)$, and extensions to general orbital angular momentum are also known [21.

Although the IR extrapolation formula (5) was derived for a single-particle degree of freedom (or systems that can be reduced to such), it has also been applied to bound states of many-body problems [19, 22, 23, 24, 25, 26] via

$$
E(L)=E_{\infty}+A_{\infty} \exp \left(-2 k_{\infty} L\right),
$$

with $L(N, \hbar \Omega)$ still to be specified. The idea is that $k_{\infty}$ can be generally interpreted as the (least) separation energy for the nucleus under consideration, so that (6) follows from the two-body derivation in Ref. [21] but now using the $S$ matrix for the corresponding break-up reaction. Because of the many approximations involved, $A_{\infty}, E_{\infty}$, and $k_{\infty}$ are all treated as fit parameters. To apply the extrapolation formula (6), one needs to work with bound states (i.e. the fully converged energy needs to be negative), $L$ must exceed the radial extent of the nucleus under consideration, and the UV cutoff $\Lambda_{\mathrm{UV}}$ must be sufficiently greater than the cutoff of the interaction. In practice, one can calculate using large values of $\hbar \Omega$ to ensure that UV corrections are much smaller than the IR corrections.

Let us consider applying Eq. (6) to compute the ground-state energies of oxygen isotopes. As a first step, we plot the energies obtained using different model spaces as a function of the effective box size $L$. The top panels in Fig. 2 show the results for ${ }^{16} \mathrm{O}$ (left) and ${ }^{24} \mathrm{O}$ (right) when plotted versus $L=L_{2}$. The data points stem from model spaces with $N=8,10,12$ oscillator quanta and $\Lambda_{\mathrm{UV}}>750 \mathrm{MeV}$, so that UV corrections are presumably small. Clearly, the data points do not fall on a single line for either nucleus, and similar results are found for ${ }^{22} \mathrm{O}$. Earlier studies [20] showed that the smoothness of $E(L)$, when plotted for a large set of $N$ and $\hbar \Omega$ values for which UV corrections are small, is a sensitive diagnostic of the quality of $L(N, \hbar \Omega)$. Thus, $L=L_{2}$ is not the accurate box size of the harmonic oscillator basis for fermionic many-body systems. In the next section we derive a more accurate value $L_{\text {eff }}$ by revisiting the underlying basis for the effective box size. As we will see, this value depends on the nucleus under consideration.

\section{Box size $L_{\text {eff }}(N, \hbar \Omega)$ for nuclei}

The paradigm for IR extrapolations in the harmonic oscillator basis can be stated as follows: For sufficiently low energies (and long wavelengths), a finite oscillator basis is indistinguishable (in the sense of an effective theory) from a spherical box with an appropriately chosen radius. This radius is determined by matching the lowest (most infrared) eigenvalue of the squared momentum operator in the oscillator basis to the lowest value in the box. To apply this matching procedure to the many-body case, we have to consider the lowest total squared momentum $\sum_{i=1}^{A} p_{i}^{2}$ for a given nucleus. To do so, we identify the occupation numbers $\nu_{n l}$ that give the lowest kinetic energy for that nucleus, and then equate the eigenvalue of the total squared momentum operator 

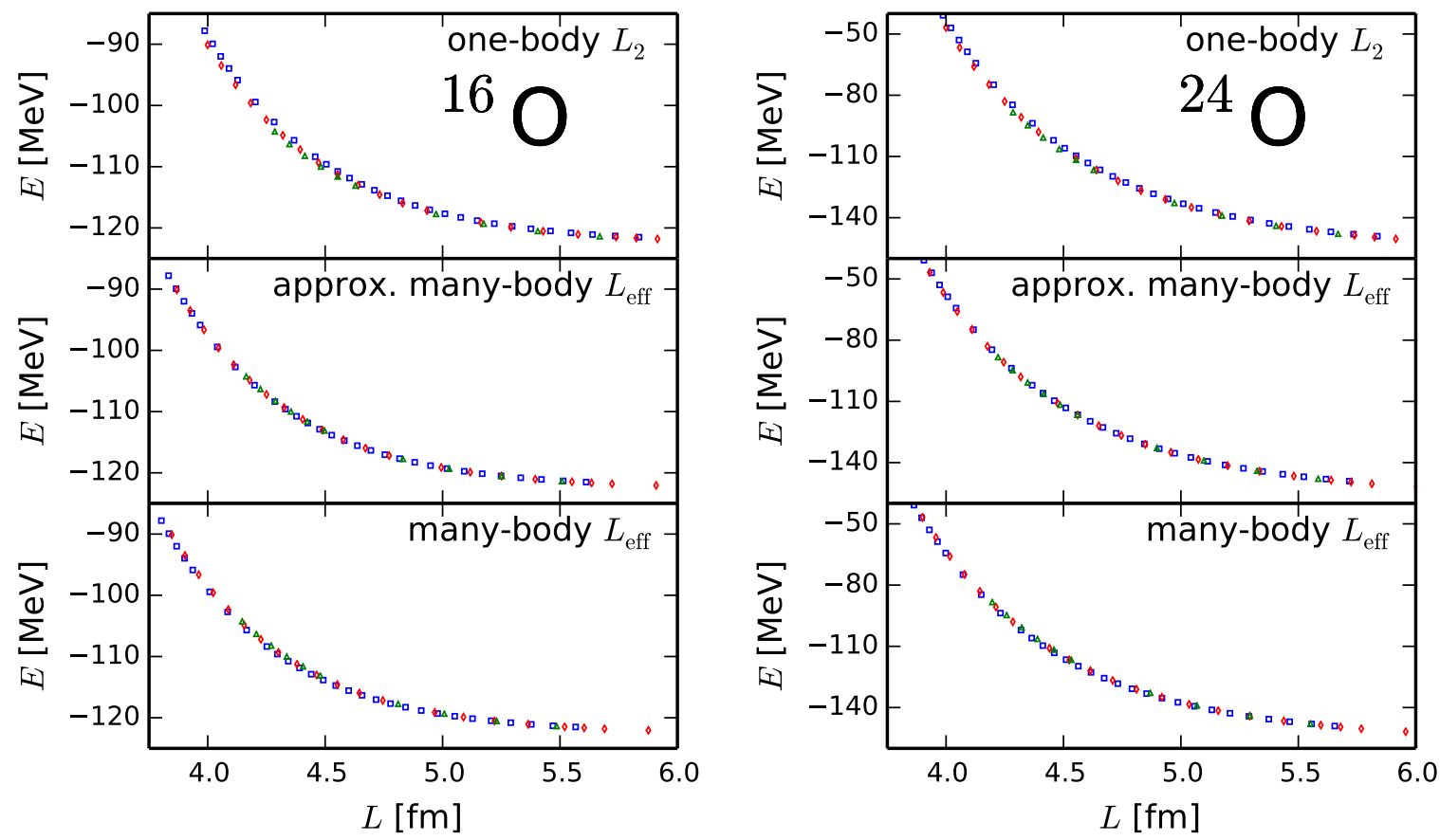

Figure 2. Ground-state energies versus the scaling variable $L$ from coupled-cluster theory in the CCSD approximation for ${ }^{16} \mathrm{O}$ (left) and ${ }^{24} \mathrm{O}$ (right) using a chiral $\mathrm{N}^{2} \mathrm{LO}$ potential with a regulator cutoff of $\Lambda_{\mathrm{UV}}=500 \mathrm{MeV}$. The squares, diamonds and triangles stem from model spaces with $N=8,10,12$, respectively. The top panels use the naive single-particle choice $L=L_{2}(N, \hbar \Omega)$, while the middle and bottom panels use approximate and exact $L=L_{\text {eff }}(N, \hbar \Omega)$, respectively, as described in Sec. 3 .

for the oscillator basis to the total squared momentum for the same number of nucleons (occupying identical partial waves) in a spherical box of size $L_{\text {eff }}$, i.e.

$$
\sum_{n l} \nu_{n l} \kappa_{l n}^{2}=\sum_{n l} \nu_{n l}\left(\frac{a_{l, n}}{L_{\mathrm{eff}}}\right)^{2} .
$$

Here, $\kappa_{l n}^{2}$ is the eigenvalue of the single-particle squared momentum operator and $a_{l, n}$ is the $(n+1)^{\text {th }}$ zero of the spherical Bessel function $j_{l}$ [27]. This yields

$$
L_{\text {eff }}=\left(\frac{\sum_{n l} \nu_{n l} a_{l, n}^{2}}{\sum_{n l} \nu_{n l} \kappa_{l n}^{2}}\right)^{1 / 2} .
$$

We can parameterize this result using $L_{2}$ by introducing $N_{\text {eff }}$, which is defined as a function of $N$ by

$$
L_{\mathrm{eff}}(N, \hbar \Omega) \equiv L_{2}\left(N_{\mathrm{eff}}, \hbar \Omega\right) .
$$

We note that the approach to the many-body scaling variable $L_{\text {eff }}$ can be extended to any localized basis set by numerically computing the eigenvalues of the total squared momentum operator and equating them to the corresponding eigenvalues of a spherical box with radius $L_{\text {eff }}$. 
For an understanding of $L_{\text {eff }}$ (or $N_{\text {eff }}$ ), it is useful to consider analytical approximations to $L_{\text {eff }}$. These are based on approximate expressions for the eigenvalues $\kappa_{l m}$ which are valid for model spaces with $N \gg 1$. In the single-particle problem (or the deuteron in its center-of-mass frame), the $(m+1)^{\text {th }}$ eigenvalue $\kappa_{l m}^{2}$ in a harmonic-oscillator model space with up to $N$ quanta of excitation is given to good approximation [21] by

$$
\kappa_{l m}^{2} \approx \frac{a_{l, m}^{2}}{2\left(N_{l}+3 / 2+2\right) b^{2}}=\frac{a_{l, m}^{2}}{\left(L_{2}(N, \hbar \Omega)\right)^{2}},
$$

and

$$
N_{l}= \begin{cases}N, & \text { for } N \text { and } l \text { even or for } N \text { and } l \text { odd; } \\ N-1, & \text { for } N \text { even and } l \text { odd or for } N \text { odd and } l \text { even. }\end{cases}
$$

As a check, consider a single particle in a model space with $N=2 n+l$. We equate the lowest eigenvalue $\kappa_{l 0}^{2}$ to the lowest eigenvalue $\left(a_{l, 0} / L\right)^{2}$ in a spherical box of radius $L$ for angular momentum $l$. This yields $L=L_{2}(N, \hbar \Omega)$ as defined in Eq. (4).

Thus, approximate values for $L_{\text {eff }}$ are found by inserting the analytical approximations (10) into Eq. (8). The middle panels of Fig. 2 show the groundstate energies for ${ }^{16,24} \mathrm{O}$ when plotted as a function of this approximate value for $L_{\text {eff }}$. Compared to the top panel $\left(L=L_{2}\right)$, the improvement is considerable and clearly visible. The bottom panels of Fig. 2 show the ground-state energies for ${ }^{16,24} \mathrm{O}$ when plotted as a function of the exact numerical value for $L_{\text {eff }}$. Again, the data points fall close to a single line. For reasons we do not yet understand, the approximate $L_{\text {eff }}$ (middle panel) leads to the smoothest line in ${ }^{16} \mathrm{O}$, while the exact $L_{\text {eff }}$ yields the smoothest line in ${ }^{24} \mathrm{O}$.

Table 1 gives $N_{\text {eff }}$ for isotopes ${ }^{12,14} \mathrm{C}$ and ${ }^{16,22,24} \mathrm{O}$. These results are determined by diagonalizing the operator $\sum_{i=1}^{A} p_{i}^{2}$ in the specified model space. We note that the "square" model space (defined by each particle allowed up to $N$ oscillator quanta) is used in the coupled-cluster calculations of this paper and by other methods [6, 7, 8]. This model space differs from the "triangular" full $N \hbar \Omega$ model space used in most NCSM calculations.

Let us make some comments on the values of $N_{\text {eff }}$ in Table 1. We recall that $N_{\text {eff }}$ is computed from the exact eigenvalues $\kappa_{l m}^{2}$ of the total squared momentum operator. The resulting values for $N_{\text {eff }}$ are a few percent smaller than the results one obtains from using the $N \gg 1$ approximation 10 in the computation of $N_{\max }$. However, these latter approximations $N_{\text {eff }}^{\text {approx }}$ can be understood semi-quantitatively as follows. For the nucleus ${ }^{4} \mathrm{He}$ (not shown) we have $N_{\text {eff }}^{\text {approx }}=N\left(N_{\text {eff }}^{\text {approx }}=N-1\right.$ ) for even (odd) $N$ because only $s$-waves are occupied, so $L_{2}(N, \hbar \Omega)=L_{\text {eff }}(N, \hbar \Omega)$. For ${ }^{12,14} \mathrm{C}$ and ${ }^{16} \mathrm{O}$, $p$-waves are at the Fermi surface, and for pure $p$-waves and even (odd) $N$ one has $N_{\text {eff }}^{\text {approx }}=N-1\left(N_{\text {eff }}^{\text {approx }}=N\right)$, see Eq. $(10)$. Thus, our values for $N_{\text {eff }}^{\text {approx }}$ are closest to these extreme values for ${ }^{16} \mathrm{O}$ and somewhat farther away for ${ }^{14,12} \mathrm{C}$. For ${ }^{22,24} \mathrm{O}, d$-shell orbitals are at the Fermi surface for the kinetic energy, countering the effects from the lower-lying $p$ waves and effectively increasing (decreasing) $N_{\text {eff }}^{\text {approx }}$ for even (odd) $N$. As 


\begin{tabular}{r|rrrrr}
\hline \hline & \multicolumn{5}{|c}{$N_{\text {eff }}$} \\
$N$ & \multicolumn{1}{|c}{${ }^{12} \mathrm{C}$} & \multicolumn{1}{c}{${ }^{14} \mathrm{C}$} & \multicolumn{1}{c}{${ }^{16} \mathrm{O}$} & ${ }^{22} \mathrm{O}$ & \multicolumn{1}{c}{${ }^{24} \mathrm{O}$} \\
\hline 1 & 0.365 & 0.393 & 0.413 & - & - \\
2 & 0.745 & 0.709 & 0.684 & 0.899 & 0.939 \\
3 & 2.515 & 2.545 & 2.566 & 1.939 & 1.844 \\
4 & 2.902 & 2.867 & 2.842 & 3.092 & 3.137 \\
5 & 4.586 & 4.617 & 4.639 & 4.116 & 4.033 \\
6 & 4.976 & 4.941 & 4.917 & 5.187 & 5.236 \\
7 & 6.629 & 6.660 & 6.682 & 6.205 & 6.127 \\
8 & 7.020 & 6.985 & 6.961 & 7.245 & 7.296 \\
9 & 8.658 & 8.689 & 8.711 & 8.259 & 8.185 \\
10 & 9.049 & 9.015 & 8.990 & 9.285 & 9.337 \\
11 & 10.678 & 10.709 & 10.732 & 10.297 & 10.225 \\
12 & 11.070 & 11.036 & 11.011 & 11.313 & 11.366 \\
13 & 12.693 & 12.725 & 12.748 & 12.324 & 12.254 \\
14 & 13.085 & 13.051 & 13.027 & 13.335 & 13.389 \\
15 & 14.705 & 14.737 & 14.760 & 14.345 & 14.276 \\
16 & 15.097 & 15.064 & 15.040 & 15.352 & 15.406 \\
17 & 16.715 & 16.747 & 16.770 & 16.361 & 16.293 \\
18 & 17.107 & 17.074 & 17.050 & 17.366 & 17.421 \\
19 & 18.723 & 18.755 & 18.778 & 18.375 & 18.307 \\
20 & 19.115 & 19.082 & 19.058 & 19.377 & 19.433 \\
\hline \hline
\end{tabular}

Table 1. Effective excitation number $N_{\text {eff }}$ for isotopes ${ }^{12,14} \mathrm{C}$ and ${ }^{16,22,24} \mathrm{O}$, computed from the exact eigenvalues of the total squared momentum operator. Where no result is given, the model space is too small to accommodate $A$ nucleons.

naively expected, the effect is larger in ${ }^{24} \mathrm{O}$ than in ${ }^{22} \mathrm{O}$. From these arguments, one expects $N-1 \leq N_{\text {eff }}^{\text {approx }} \leq N$ in general.

We note that the defect $N-N_{\text {eff }}$ varies very little (focusing on either even or odd $N)$ as $N$ varies. In practice, one might thus take a fixed defect (say from $N=10$ or so) and consider the small variation of the defect a higher-order correction. We also note an odd-even staggering of $N_{\text {eff }}$. This staggering is caused by Eq. (11) and has its root in the fact that $L_{2}$ increases for even (odd) values of angular momentum only at even (odd) values of $N$. In most NCSM and coupled-cluster calculations, practitioners limit themselves to sequences of model spaces with either even or odd values of $N$.

\section{Extrapolations and the coupled-cluster method}

In this section, we apply IR extrapolations to the results of the coupled-cluster method with singles and doubles (CCSD) calculations [3, 4, 28, 5]. Our interest is twofold. First, we want to study in more detail whether $L_{\text {eff }}$ is also the relevant length scale when 
approximate many-body solutions (such as CCSD) are employed. We note that most $a b$ initio methods that presently compute nuclei beyond the $p$ shell employ approximate solutions of the many-body problem [29, 30, 7, 25, 24]. Second, we want to probe the extrapolation formula (6) over a large energy range and see how large the model space needs to be for a reliable and accurate extrapolation.

We compute the ground-state energies of the nuclei ${ }^{16,22,24} \mathrm{O}$ using the nextto-next-to-leading order chiral nucleon-nucleon interaction of Ref. [9] with a cutoff $\Lambda_{\chi}=500 \mathrm{MeV}$. This interaction has been optimized to scattering data of the nucleonnucleon system and deuteron bound-state properties. It is similar in quality to the chiral nucleon-nucleon interaction $\mathrm{NNLO}_{\text {opt }}$ [31], which was optimized with respect to phase shifts.

Figure 3 shows the CCSD ground state energies for ${ }^{16} \mathrm{O}$ as a function of $L_{\text {eff }}$ (left figure) and $\hbar \Omega$ (right figure) for model spaces with $N=8,10,12$. Our model spaces have oscillator frequencies in the interval $\hbar \Omega /(\mathrm{MeV}) \in[16,70]$. At fixed $N$, the energy computed at the highest oscillator frequency corresponds to the smallest value of $L_{\text {eff }}$ and exhibits the smallest UV error. In the left panel of Fig. 3 the solid data points forming the exponential envelope have negligible UV corrections and can be used in the IR extrapolation. As seen in the right panel, these frequencies are much larger than the naive estimate $\hbar \Omega_{\min } \approx \hbar^{2} \Lambda_{\chi} /(m R)$ that minimizes the energy for a nucleus of radius $R$ and interaction with cutoff $\Lambda_{\chi}$ [17]. The exponential extrapolation (6) to all solid data points is shown as a dashed line, and the asymptote $E_{\infty}$ as a full line.

The extrapolation results are from a least-squares penalty-function fit with equal weighting [32] of the parameters $E_{\infty}, k_{\infty}$, and $A_{\infty}$. We note that the exponential extrapolation practically works over the entire range of about $60 \mathrm{MeV}$. While the energy correction can be a substantial fraction of the total binding energy, we have $\exp \left(-2 k_{\infty} L_{\text {eff }}\right) \ll 1$ over the considered range of $L_{\text {eff }}$. For fixed $N$, the (very small) UV corrections are expected to increase with increasing $L_{\text {eff }}$, see Fig. 20 of Ref. [20] as an example. We note that all solid data points employed in the IR extrapolation in Fig. 3 are from model spaces with $\Lambda_{\mathrm{UV}}>750 \mathrm{MeV}$. The UV cutoff parameter of the nucleon-nucleon interaction used here is $\Lambda_{\chi} \approx 500 \mathrm{MeV}$, but this is not a sharp cutoff, which is why $\Lambda_{\mathrm{UV}}$ must be sufficiently higher than $\Lambda_{\chi}$ so that effects from the omitted UV tail are small.

Let us discuss errors and error estimates. We applied a theory, derived for the deuteron and $k_{\infty} L_{\text {eff }} \gg 1$ to many-body systems and for $k_{\infty} L_{\text {eff }}>4$ or so. We view the extrapolation formula (6) as a leading order and systematically improvable result applied to a complex nucleus over a wide range of $k_{\infty} L_{\text {eff }}$, neglecting higher-order corrections. We also deal with systematic errors from the CCSD approximation (recall that $L_{\text {eff }}$ was worked out for an exact solution of the operator $\sum_{i=1}^{A} p_{i}^{2}$ ). We believe that UV errors are negligible. Thus, we have systematic errors from neglected corrections in the IR and from the approximate many-body method. Both systematic errors are hard to quantify. Some aspects of these systematic errors behave as statistical errors. For instance, we deal with a relatively small scatter of our data points around the exponential extrapolation. These 

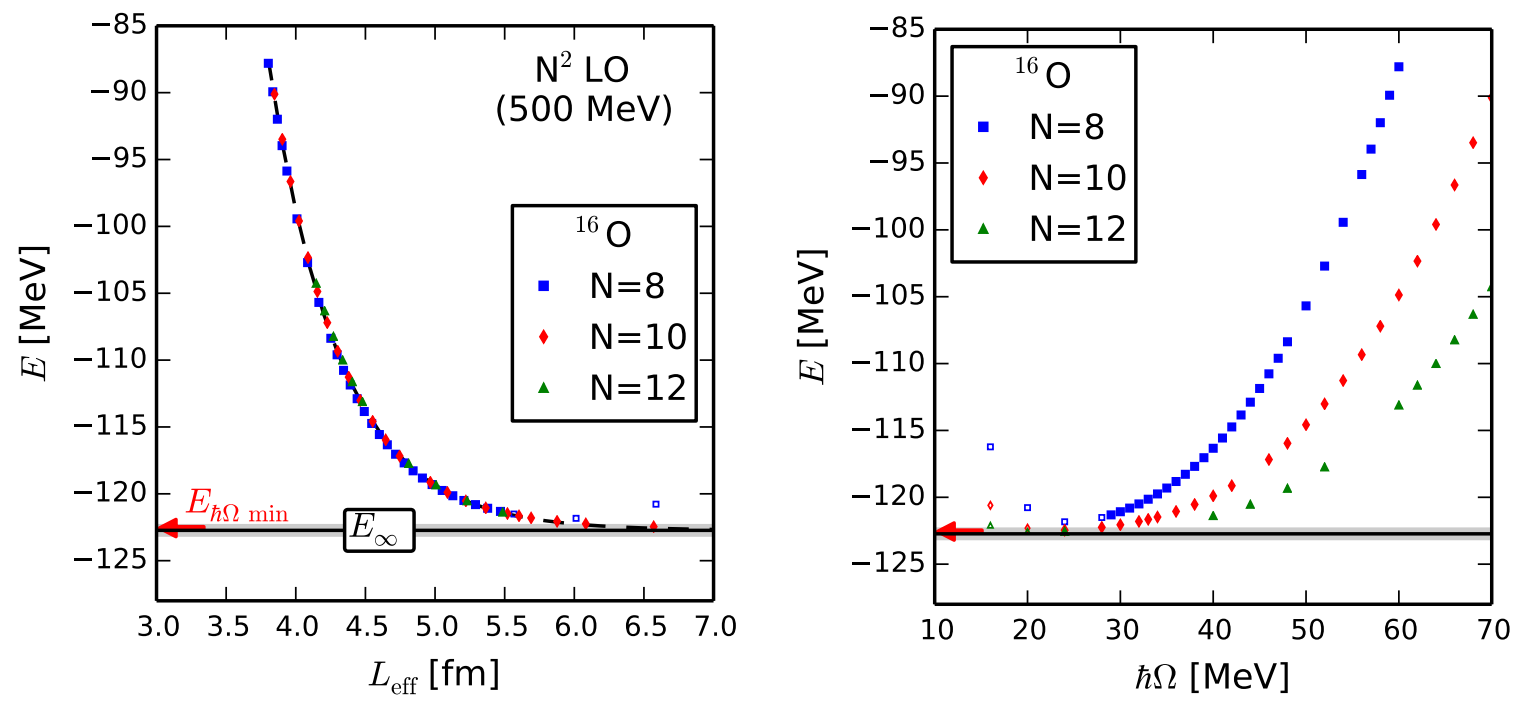

Figure 3. Ground-state energies in CCSD approximation for ${ }^{16} \mathrm{O}$ as a function of $L_{\text {eff }}$ (left panel) and $\hbar \Omega$ (right panel) for harmonic-oscillator spaces with $N$ as indicated. Dashed line: exponential fit of Eq. (5) to data with $N_{\max }=12$. Full line with band: asymptote $E_{\infty}$ from fit with $95 \%$ confidence interval. Hollow markers: points excluded from fit. The arrow marks the minimum energy $E_{\hbar \Omega \text { min }}$ that is obtained when varying the oscillator spacing $\hbar \Omega$ for $N_{\max }=12$.

(relatively small) errors are easy to quantify and usually returned by fitting routines in the form of standard asymptotic errors computed from the covariance matrix. At this moment, these are the only errors we quantify and present in tables below. However, we emphasize that these errors are presumably much smaller than the systematic errors.

We fit Eq. (6) to ${ }^{16} \mathrm{O}$, including increasingly larger sets of data points from model spaces with $N \leq N_{\max }$. Table 2 shows the results. The error estimates are from the covariance matrix. We repeat that any systematic errors from sub-leading IR corrections or due to the CCSD approximation are not included. We note that the $E_{\infty}$ result for $N_{\max }=8$ is within the error estimates for $N_{\max }=10,12$. Table 2 also shows the minimum energy $E_{\hbar \Omega \text { min }}$ that is obtained at fixed $N_{\max }$ when varying the oscillator spacing $\hbar \Omega$. For the smaller model space with $N_{\max }=8$, the extrapolated energy $E_{\infty}$ is much closer to the "true" result (from extrapolations in larger model spaces) than the minimum energy $E_{\hbar \Omega \min }$, and this is the practical value of IR extrapolations. Of course, the challenge remains to give a meaningful error estimate for all model spaces.

The left panel of Fig. 4 shows a log plot of the difference $\Delta E=E-E_{\infty}$ for ${ }^{16} \mathrm{O}$. The dashed line is the $N_{\max }=12$ exponential fit from Table 2. Deviations at the largest values of $L_{\text {eff }}$ reflect UV corrections (open symbols are not used in the fit) and other systematic errors discussed above. However, the consistency of the fit to Eq. (6) over a large range of $\Delta E$ is striking. This suggests that there is enough information for reliable extrapolations even when using only calculations far from the energy minimum. This is validated for ${ }^{16} \mathrm{O}$ in the right panel of Fig. 4, where extrapolations are shown for 
Infrared extrapolations for atomic nuclei

\begin{tabular}{lrrr}
\hline \hline \multicolumn{1}{c}{$N_{\max }$} & \multicolumn{1}{c}{8} & \multicolumn{1}{c}{10} & \multicolumn{1}{c}{12} \\
\hline$E_{\hbar \Omega \min }[\mathrm{MeV}]$ & -121.83 & -122.46 & -122.56 \\
$E_{\infty}[\mathrm{MeV}]$ & $-122.62 \pm 0.06$ & $-122.68 \pm 0.35$ & $-122.73 \pm 0.35$ \\
$k_{\infty}\left[\mathrm{fm}^{-1}\right]$ & $1.00 \pm 0.00$ & $0.99 \pm 0.01$ & $0.98 \pm 0.01$ \\
$A_{\infty}\left[10^{4} \mathrm{MeV}\right]$ & $6.95 \pm 0.20$ & $6.48 \pm 0.49$ & $5.96 \pm 0.63$ \\
\hline \hline
\end{tabular}

Table 2. Extrapolation parameters (and statistical error estimates) and energy minima for ${ }^{16} \mathrm{O}$ as a function of basis truncation $N_{\max }$. The neglected systematic errors are expected to dominate the error budget.

fixed $N=8,10$, and 12 using only points in each case with $\hbar \Omega \geq 50 \mathrm{MeV}$. This plot can also be interpreted as showing that controlled and consistent (if not highly precise) extrapolations to $\hbar \Omega=0$ (i.e. removing the IR cutoff for a fixed $N$ ) can be achieved if UV corrections are suppressed.
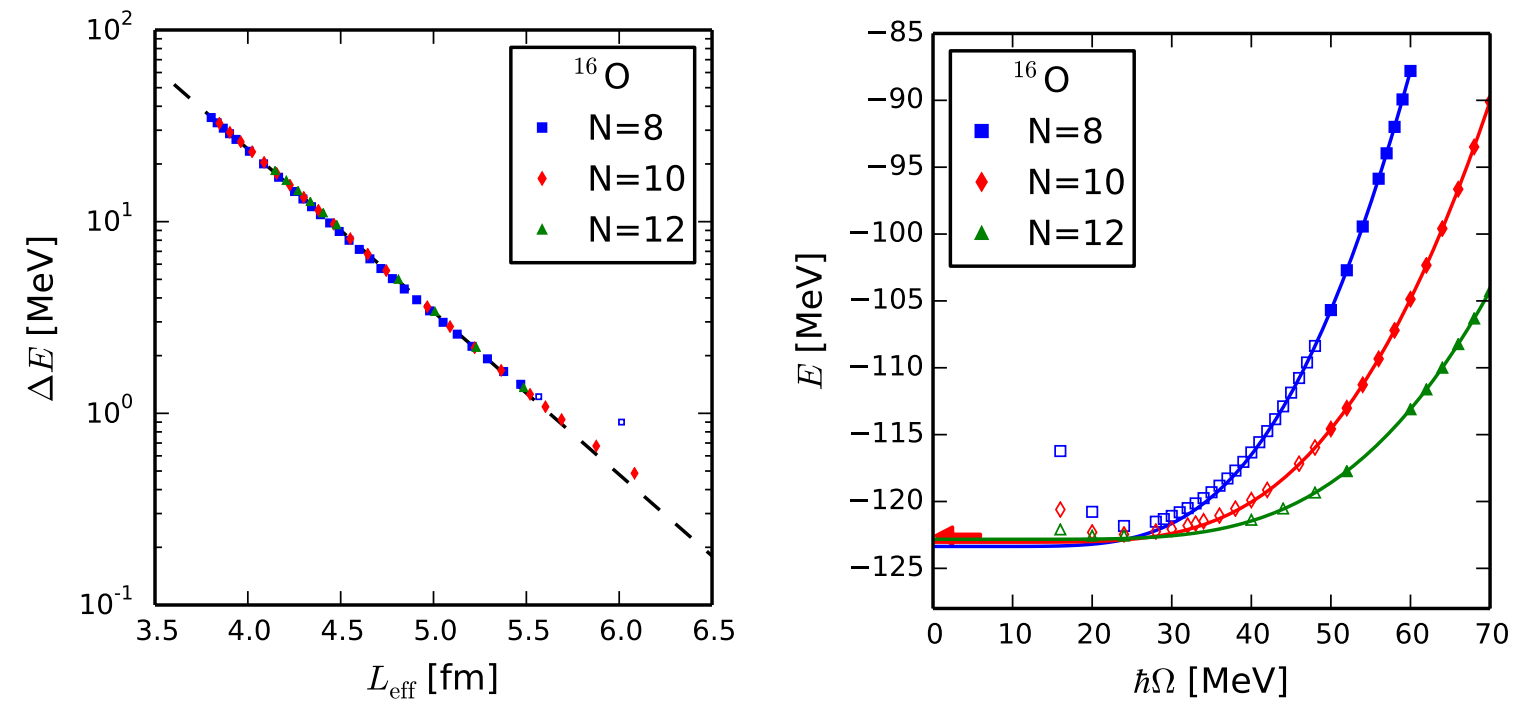

Figure 4. Left: energy difference $\Delta E$ for the ground-state energy (in CCSD approximation) of ${ }^{16} \mathrm{O}$ as a function of $L_{\text {eff }}$ in a log plot. The symbols are as in Fig. 3 and the dashed line is the exponential fit for $N_{\max }=12$ from Table 2. Right: ground-state energies as a function of $\hbar \Omega$ as in the right panel of Fig. 3. The solid lines are fits of Eq. 6 for fixed $N$ to only the solid points. The arrow marks the minimum energy $E_{\hbar \Omega \text { min }}$ that is obtained when varying the oscillator spacing $\hbar \Omega$ for $N_{\max }=12$.

Now we turn to the neutron-rich isotopes ${ }^{22,24}$ O. Figure 5 shows the CCSD groundstate energies for ${ }^{22} \mathrm{O}$ (left) and ${ }^{24} \mathrm{O}$ (right) as a function of $L_{\text {eff }}$. The exponential extrapolation (6), and the extrapolated ground-state energies $E_{\infty}=-148.41 \pm 0.60 \mathrm{MeV}$ (for ${ }^{22} \mathrm{O}$ ) and $E_{\infty}=-155.38 \pm 0.83 \mathrm{MeV}$ (for ${ }^{24} \mathrm{O}$ ) are also shown. These results employ model spaces with $N=8,10,12$. Table 3 shows the extrapolated energies (and error estimates from the $\chi^{2}$ fit) when only data points with $N \leq N_{\max }$ are employed in the 

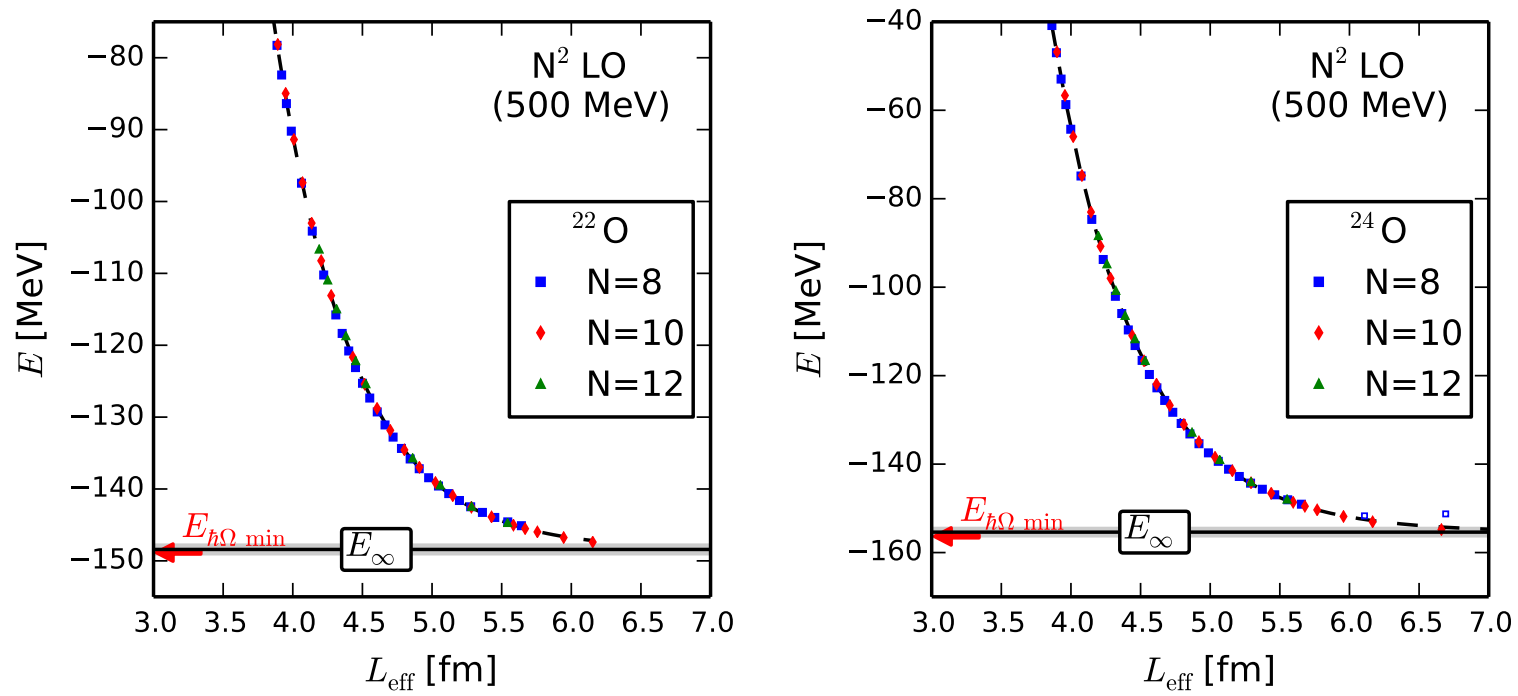

Figure 5. (Color online) Ground-state energies (CCSD approximation) for ${ }^{22} \mathrm{O}$ (left) and ${ }^{24} \mathrm{O}$ (right) as a function of $L_{2}$ for harmonic oscillator spaces with $N$ as indicated. Dashed line: Exponential fit to Eq. (6). Full line with band: Asymptote $E_{\infty}$ from errors from covariance matrix. Hollow markers: Points excluded from fit. The arrow marks the minimum energy $E_{\hbar \Omega \text { min }}$ that is obtained when varying the oscillator spacing $\hbar \Omega$ for $N_{\max }=12$.

fit. We note that the fits work well over an energy range of tens of $\mathrm{MeVs}$ (see the left panels of Figs. 6 and 7). Again, the value of the IR extrapolation lies in the finding that $N_{\text {max }}=8$ extrapolations yield results that are close to the "true" ground-state energies (see the right panels of Figs. 6 and 7).

\begin{tabular}{llrrr}
\hline \hline & \multicolumn{1}{c}{$N_{\max }$} & \multicolumn{1}{c}{8} & \multicolumn{1}{c}{10} & \multicolumn{1}{c}{12} \\
\hline \multirow{2}{*}{${ }^{22} \mathrm{O}$} & $E_{\infty}[\mathrm{MeV}]$ & $-147.93 \pm 0.01$ & $-148.27 \pm 0.47$ & $-148.41 \pm 0.60$ \\
& $k_{\infty}\left[\mathrm{fm}^{-1}\right]$ & $0.91 \pm 0.00$ & $0.90 \pm 0.01$ & $0.89 \pm 0.00$ \\
& $A_{\infty}\left[10^{4} \mathrm{MeV}\right]$ & $8.56 \pm 0.02$ & $7.76 \pm 0.52$ & $7.19 \pm 0.33$ \\
\hline \multirow{2}{*}{${ }^{24} \mathrm{O}$} & $E_{\infty}[\mathrm{MeV}]$ & $-154.42 \pm 0.10$ & $-155.08 \pm 0.63$ & $-155.38 \pm 0.83$ \\
& $k_{\infty}\left[\mathrm{fm}^{-1}\right]$ & $0.83 \pm 0.00$ & $0.83 \pm 0.01$ & $0.82 \pm 0.00$ \\
& $A_{\infty}\left[10^{4} \mathrm{MeV}\right]$ & $7.89 \pm 0.11$ & $7.06 \pm 0.52$ & $6.53 \pm 0.31$ \\
\hline \hline
\end{tabular}

Table 3. Extrapolated energies $E_{\infty}$ for ${ }^{22,24} \mathrm{O}$ as a function basis truncation $N_{\max }$. The energy minima for ${ }^{22,24} \mathrm{O}$ in a $N_{\max }=12$ model-space are found at the oscillator frequency $\hbar \Omega=20 \mathrm{MeV}$ and are $E_{\hbar \Omega \text { min }}=-148.85 \mathrm{MeV}$ and $E_{\hbar \Omega \min }=-156.35 \mathrm{MeV}$, respectively.

Our study shows that IR extrapolation can be a practical tool for approximate solutions of the nuclear many-body problem. The extrapolation is accurate and reliable over a large energy range of tens of $\mathrm{MeV}$. For the employed chiral interaction at NNLO, the examples of ${ }^{16,22,24} \mathrm{O}$ suggest that ground-state energies of $p$-shell and $s d$-shell nuclei 

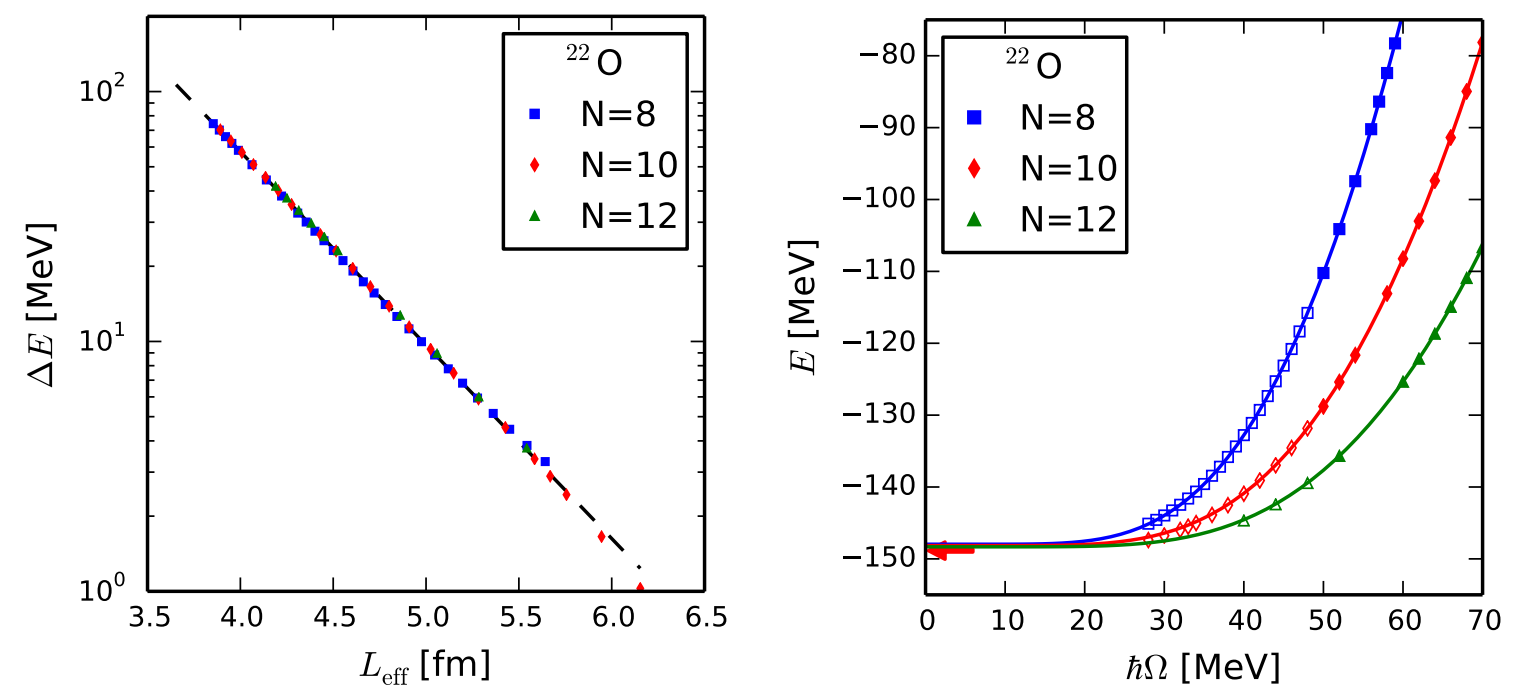

Figure 6. Left: energy difference $\Delta E$ for the ground-state energy (in CCSD approximation) of ${ }^{16} \mathrm{O}$ as a function of $L_{\text {eff }}$ in a $\log$ plot. The symbols are as in Fig. 3, and the dashed line is the exponential fit for $N_{\max }=12$ from Table 3 . Right: ground-state energies as a function of $\hbar \Omega$ as in the right panel of Fig. 3 . The solid lines are fits of Eq. (6) for fixed $N$ to only the solid points. The arrow marks the minimum energy $E_{\hbar \Omega \text { min }}$ that is obtained when varying the oscillator spacing $\hbar \Omega$ for $N_{\max }=12$.
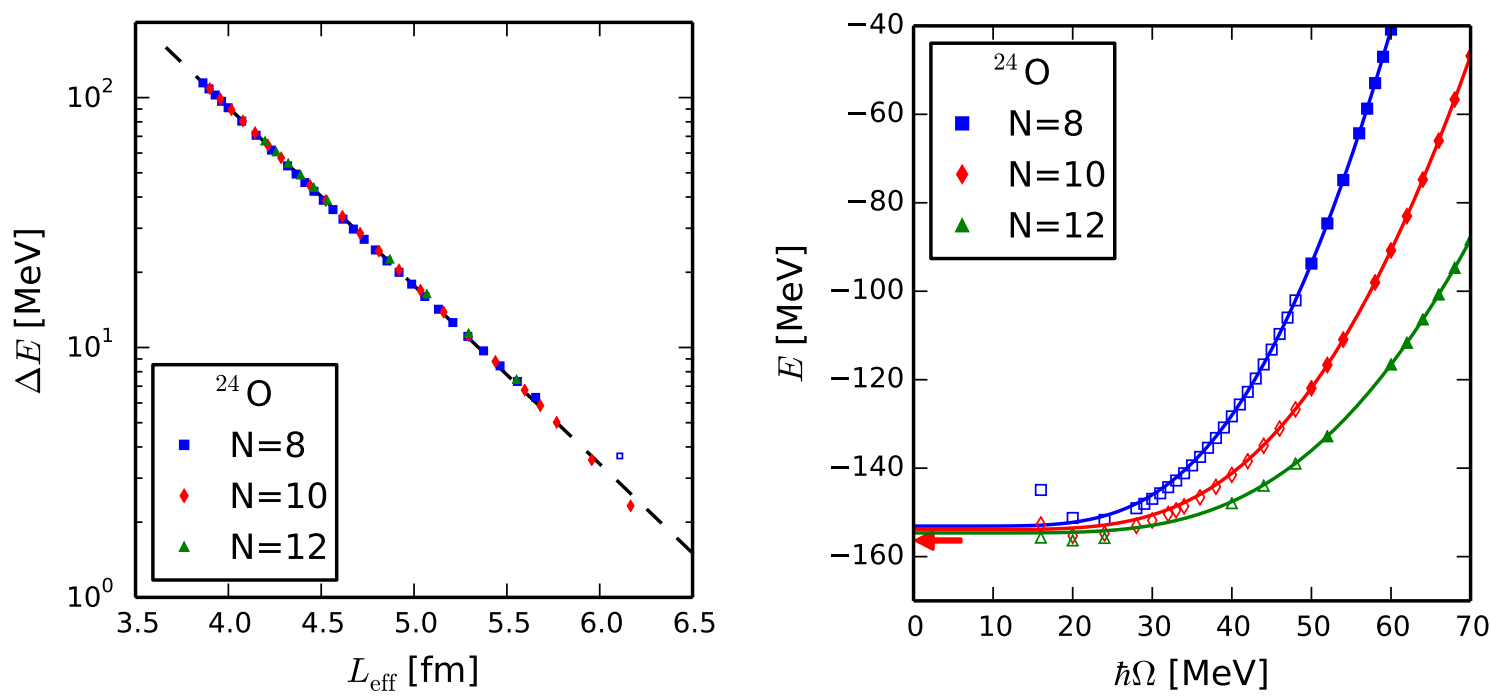

Figure 7. Left: energy difference $\Delta E$ for the ground-state energy (in CCSD approximation) of ${ }^{24} \mathrm{O}$ as a function of $L_{\text {eff }}$ in a $\log$ plot. The symbols are as in Fig. 3, and the dashed line is the exponential fit for $N_{\max }=12$ from Table 3. Right: ground-state energies as a function of $\hbar \Omega$ as in the right panel of Fig. 3. The solid lines are fits of Eq. 6 for fixed $N$ to only the solid points. The arrow marks the minimum energy $E_{\hbar \Omega \text { min }}$ that is obtained when varying the oscillator spacing $\hbar \Omega$ for $N_{\max }=12$. 
can be extrapolated from model spaces with $N=8$.

We also tried to include $N=6$ data points in the extrapolation, but the data points did not fall onto the same line as the $N=8,10,12$ points. We speculate that this is due to peculiarities of the CCSD approximation, or due to a less complete decoupling of the center of mass in small model spaces [17. Again, this points to the need to better understand the systematic errors involved in IR extrapolations of results obtained with approximate many-body results.

\section{Summary}

We studied IR extrapolations for coupled-cluster computations of oxygen isotopes with chiral nucleon-nucleon interactions at NNLO. One of the main results is the identification of the nucleus-dependent infrared box size $L_{\text {eff }}$. Our results show that IR extrapolations are feasible in practice, but we need a better understanding of systematic errors for extrapolated quantities; a Bayesian framework may be useful in this regard. Nevertheless, we demonstrated that reliable IR extrapolations can be performed in $p-s-d$ nuclei spanning over tens of MeVs. For the IR extrapolations to work in practice one needs to minimize UV corrections and work at frequencies away from the usual range about the oscillator frequency that minimizes the energy at fixed number $N$ of oscillator quanta.

\section{Acknowledgments}

This material is based upon work supported in part by the National Science Foundation under Grant No. PHY-1306250 (Ohio State University), by the U.S. Department of Energy, Office of Science, Office of Nuclear Physics, under Award Numbers DEFG02-96ER40963 (University of Tennessee), de-sc0008499/DE-SC0008533 (SciDAC-3 NUCLEI Collaboration), the Field Work Proposal ERKBP57 at Oak Ridge National Laboratory, and under contract number DEAC05-00OR22725 (Oak Ridge National Laboratory).

\section{References}

[1] Navrátil P, Quaglioni S, Stetcu I and Barrett B R 2009 Journal of Physics G: Nuclear and Particle Physics 36083101 URL http://stacks.iop.org/0954-3899/36/i=8/a=083101

[2] Barrett B R, Navrátil P and Vary J P 2013 Progress in Particle and Nuclear Physics 69131 181 URL http://www.sciencedirect.com/science/article/pii/S0146641012001184

[3] Kümmel H, Lührmann K H and Zabolitzky J G 1978 Physics Reports 361 - 63 URL http: //www.sciencedirect.com/science/article/pii/0370157378900819

[4] Bishop R F 1991 Theoretical Chemistry Accounts: Theory, Computation, and Modeling (Theoretica Chimica Acta) 80(2) 95-148 10.1007/BF01119617 URL http://dx.doi.org/10. $1007 /$ BF01119617

[5] Hagen G, Papenbrock T, Hjorth-Jensen M and Dean D J 2013 ArXiv:1312.7872 (Preprint 1312.7872) URL http://adsabs.harvard.edu/abs/2013arXiv1312.7872H 
[6] Dickhoff W and Barbieri C 2004 Progress in Particle and Nuclear Physics 52377 - 496 URL http://www.sciencedirect.com/science/article/pii/S0146641004000535

[7] Tsukiyama K, Bogner S K and Schwenk A 2011 Phys. Rev. Lett. 106(22) 222502 URL http: //link.aps.org/doi/10.1103/PhysRevLett.106.222502

[8] Hergert H, Binder S, Calci A, Langhammer J and Roth R 2013 Phys. Rev. Lett. 110(24) 242501 URL http://link.aps .org/doi/10.1103/PhysRevLett.110.242501

[9] Ekström A, Jansen G R, Wendt K A, Hagen G, Papenbrock T, Bacca S, Carlsson B and Gazit D 2014 ArXiv:1406.4696 URL http://adsabs.harvard.edu/abs/2014arXiv1406.4696E

[10] Michel N, Nazarewicz W, Płoszajczak M and Vertse T 2009 Journal of Physics G: Nuclear and Particle Physics 36013101 URL http://stacks.iop.org/0954-3899/36/i=1/a=013101

[11] Caprio M A, Maris P and Vary J P 2012 Phys. Rev. C 86(3) 034312 URL http://link.aps. org/doi/10.1103/PhysRevC.86.034312

[12] Bulgac A and Forbes M M 2013 Phys. Rev. C 87(5) 051301 URL http://link.aps.org/doi/ 10.1103/PhysRevC.87.051301

[13] Bogner S K, Kuo T T S and Schwenk A 2003 Physics Reports 3861 - 27 URL http: //www.sciencedirect.com/science/article/pii/S0370157303002953

[14] Bogner S K, Furnstahl R J and Perry R J 2007 Phys. Rev. C 75(6) 061001 URL http: //link.aps.org/doi/10.1103/PhysRevC.75.061001

[15] Bohigas O, Tomsovic S and Ullmo D 1993 Physics Reports 22343 - 133 ISSN 0370-1573 URL http://dx.doi.org/10.1016/0370-1573(93)90109-Q

[16] Stetcu I, Barrett B and van Kolck U 2007 Physics Letters B 653358 - 362 URL http: //www.sciencedirect.com/science/article/pii/S0370269307009185

[17] Hagen G, Papenbrock T, Dean D J and Hjorth-Jensen M 2010 Phys. Rev. C 82(3) 034330 URL http://link.aps.org/doi/10.1103/PhysRevC.82.034330

[18] Coon S A, Avetian M I, Kruse M K G, van Kolck U, Maris P and Vary J P 2012 Phys. Rev. C 86(5) 054002 URL http://link.aps.org/doi/10.1103/PhysRevC.86.054002

[19] Furnstahl R J, Hagen G and Papenbrock T 2012 Phys. Rev. C 86(3) 031301 URL http: //link.aps.org/doi/10.1103/PhysRevC.86.031301

[20] More S N, Ekström A, Furnstahl R J, Hagen G and Papenbrock T 2013 Phys. Rev. C 87(4) 044326 URL http://link .aps .org/doi/10.1103/PhysRevC.87.044326

[21] Furnstahl R J, More S N and Papenbrock T 2014 Phys. Rev. C 89(4) 044301 URL http: //link.aps.org/doi/10.1103/PhysRevC.89.044301

[22] Jurgenson E D, Maris P, Furnstahl R J, Navrátil P, Ormand W E and Vary J P 2013 Phys. Rev. C 87(5) 054312 URL http://link.aps.org/doi/10.1103/PhysRevC.87.054312

[23] Roth R, Calci A, Langhammer J and Binder S 2013 ArXiv:1311.3563 URL http://adsabs. harvard.edu/abs/2013arXiv1311.3563R

[24] Somà V, Barbieri C and Duguet T 2013 Phys. Rev. C 87(1) 011303 URL http://link.aps .org/ doi/10.1103/PhysRevC.87.011303

[25] Hergert H, Bogner S K, Binder S, Calci A, Langhammer J, Roth R and Schwenk A 2013 Phys. Rev. C 87(3) 034307 URL http://link.aps.org/doi/10.1103/PhysRevC.87.034307

[26] Sääf D and Forssén C 2014 Phys. Rev. C 89(1) 011303 URL http://link.aps.org/doi/10. 1103/PhysRevC.89.011303

[27] Olver F W J, Lozier D W, Boisvert R F and Clark C W (eds) 2010 NIST Handbook of Mathematical Functions (New York, NY: Cambridge University Press)

[28] Bartlett R J and Musiał M 2007 Rev. Mod. Phys. 79(1) 291-352 URL http://link.aps.org/ doi/10.1103/RevModPhys.79.291

[29] Hagen G, Papenbrock T, Dean D J and Hjorth-Jensen M 2008 Phys. Rev. Lett. 101(9) 092502 URL http://link.aps.org/doi/10.1103/PhysRevLett.101.092502

[30] Barbieri C and Hjorth-Jensen M 2009 Phys. Rev. C 79(6) 064313 URL http://link.aps.org/ doi/10.1103/PhysRevC.79.064313

[31] Ekström A, Baardsen G, Forssén C, Hagen G, Hjorth-Jensen M, Jansen G R, Machleidt R, 
Nazarewicz W, Papenbrock T, Sarich J and Wild S M 2013 Phys. Rev. Lett. 110(19) 192502 URL http://link .aps .org/doi/10.1103/PhysRevLett.110.192502

[32] Dobaczewski J, Nazarewicz W and Reinhard P G 2014 Journal of Physics G: Nuclear and Particle Physics 41074001 URL http://stacks .iop.org/0954-3899/41/i=7/a=074001 